\title{
UNIQUENESS OF VERY SINGULAR SELF-SIMILAR SOLUTION OF A QUASILINEAR DEGENERATE PARABOLIC EQUATION WITH ABSORPTION
}

\author{
J.I. DIAZ* AND J.E. SAA*
}

Abstract

We show the uniqueness of the very singular self-similar solution of the equation

$$
u_{t}-\Delta_{p} u^{m}+u^{q}=0 .
$$

The result is carried out by studying the stationary associate equation and by introducing a suitable change of unknown. That allows to assume the zero-order perturbation term in the new equation to be monotone increasing. A careful study of the behaviour of solutions near the boundary of their support is also used in order to prove the main result.

\section{Introduction}

The main goal of this paper is to show the uniqueness of solutions of the following quasilinear elliptic problem

(1) $-\left(\left|u^{\prime}\right|^{p-2} u^{\prime}\right)^{\prime}-\frac{(N-1)}{x}\left|u^{\prime}\right|^{p-2} u^{\prime}+h\left(x, u, u^{\prime}\right)=g(x, u), \quad x>0$,

$$
u^{\prime}(0)=0, \quad \lim _{x \rightarrow \infty} u(x)=0
$$

$$
u(x) \geq 0 \quad(\not \neq 0)
$$

"Partially supported by the DGICYT project n" PB90/0620. 
in which $p>1$ and the functions $h$ and $g$ satisfy certain structural conditions which will be made explicit later.

The rnain motivation for the consideration of such a problem comes from the study of very singular solutions of the quasilinear degenerate parabolic equation with absorption

$$
u_{t}=\Delta_{p} u^{m}-u^{q} \quad \text { in } \quad Q=\mathbb{R}^{N} \times(0, \infty)
$$

where, as usual, $\Delta_{p} u$ denotes the $p$-Laplacian operator

$$
\Delta_{p} v=\operatorname{div}\left(|\nabla v|^{p-2} \nabla v\right), \quad 1<p<\infty,
$$

$N \geq 1$ and $m$ and $q$ are nonnegative real numbers. Equation (4) contains, as special cases, the equations

$$
u_{t}=\Delta u^{m}-u^{q}
$$

and

$$
u_{t}=\Delta_{p} u-u^{q}
$$

which have been intensively studied in the last years. For many different purposes it is interesting to study singular solutions of (4) i.e. nonnegative functions $u$ satisfying (4) in $Q$ (in the sense of distributions) and such that $u(x, 0)=0$ if $x \in \mathbb{R}^{N}-\{0\}$. In many cases, the singularity at $t=0$ of such a solution must be as that of the fundomental solution i.e.

$$
u(x, 0)=c \delta(x)
$$

for somc positive constant $c$, or, in other words,

$$
\lim _{t \rightarrow 0} \int_{|x|<r} u(x, t) d x=c
$$

for any $r>0$. Nevertheless, when the absorption is strong enough with respect to the diffusion, there exists another type of singular solution $u$ called as very singular solution which has been discovered previously in the following cases:

a) equation (5) with $m=1$ and $1<q<1+(2 / N)$ : Brezis, Peletier and Terman $[\mathbf{1}]$

b) equation (5) with $m>1$ and $m<q<m+(2 / N)$ : Peletier and Terman $[7]$

c) equation (6) with $p>q$ and $p-1<q<p-1+(p / N)$ : Peletier and Wang $[8]$. 
In all those cases this new singular solution satisfies

$$
\lim _{t \rightarrow 0} \int_{|x|<r} u(x, t) d x=+\infty
$$

for any $r>0$ and so it is more singular than the fundamental solution. As usual, the existence of a very singular solution is obtained in the class of self-similar solutions

$$
W(x, t)=t^{-1 /(q-1)} f\left(|x| / t^{1 / \beta}\right)
$$

where $\beta$ must be suitable chosen. For instance $\beta=2(q-1) /(q-m)$ and $\beta=p(q-1) /(q+1-p)$ in the cases of equations (5) and (6) respectively (recall that $q>p-1$ ). More generally, we can consider self-similar solutions $W$ of the equation (4) in which case the natural choice of $\beta$ is

$$
\beta=p(q-1) /(q-m(p-1))
$$

A function $W$ given by (8) is then a very singular solution if $f$ satisfics

$$
\begin{array}{r}
\left(\left|\left(f^{m}\right)^{\prime}\right|^{p-2}\left(f^{m}\right)^{\prime}\right)^{\prime}+\frac{(N-1)}{x}\left|\left(f^{m}\right)^{\prime}\right|^{p-2}\left(f^{m}\right)^{\prime}+\frac{1}{\beta} x f^{\prime}+\frac{1}{(q-1)} f-f^{q}= \\
=0, \text { in }(0, \infty)
\end{array}
$$

$$
\begin{gathered}
f \geq 0 \text { in }(0, \infty) \\
f^{\prime}(0)=0, \quad \lim _{n \rightarrow \infty} \eta^{p} /(\eta-n(p-1)) f(\eta)=0
\end{gathered}
$$

The uniqueness of $f$ solution of (11) (12) (13) was only given for the case $m=1$ and $p=2$, (see [1]) and was left open in [7] and [8]. The main goal of our work is to give an uniqueness result true for any value of $m$ and $p$.

Introducing $v=f^{m}$, we remark that $v$ satisfies an equation of the type (1) with

$$
g(x, u)=-u^{q / m}+\frac{1}{(q-1)} u^{1 / m}
$$

and

$$
h(x, u)=-\frac{1}{m \beta} x u^{-\frac{(m-1)}{m}} u^{\prime}
$$

So $g(x, u)$ is not monotone in $u$. Moreover the differential terms in equation (11) may have different homogeneity $(m(p-1)$ and 1 respectively) which leads to some special difficulties (solutions with compact support if $m(p-1)>1$, etc $)$. 


\section{The main results}

We shall prove the uniqueness of solutions of the problem

$$
\begin{gathered}
\left(\left|\left(u^{m}\right)^{\prime}\right|^{p-2}\left(u^{m}\right)^{\prime}\right)^{\prime}+\frac{(N-1)}{x}\left|\left(u^{m}\right)^{\prime}\right|^{p-2}\left(u^{m}\right)^{\prime}+\frac{1}{\beta} x u^{\prime}+G(u)=0, \\
u(x) \geq 0 \quad(\not \equiv 0) \\
\left(u^{m}\right)^{\prime}(0)=0, \quad \lim _{x \rightarrow \infty} u(x)=0
\end{gathered}
$$

where $m>0, p>1, N \geq 1, \beta>0$ and

$$
G(u)=\frac{1}{q-1} u-u^{q} .
$$

For some values of $m$ and $p$ problem (14) (15) (16) does not have any classical solution and it must be solved in a generalized way. This is the case when $m(p-1)>1$ because the solutions have as support a compact interval $\left[0, x_{0}\right]$ and $u^{\prime}$ may be discontinuous at $x=x_{0}$ (see part (v) of Lemma 1). To define the notion of weak solution we multiply the equation (14) by a smooth test function $\xi(x)$ with compact support in $[0, \infty)$ but not necessarily vanishing at $x=0$. By multiplying by $x^{N-1}$ and integrating by parts we obtain

$$
\begin{aligned}
-\int_{0}^{\infty} x^{N-1}\left|\left(u^{m}\right)^{\prime}\right|^{p-2}\left(u^{m}\right)^{\prime} \xi^{\prime} d x & -\frac{1}{\beta} \int_{0}^{\infty} x^{N} u \xi^{\prime} d x+ \\
& +\int_{0}^{\infty} x^{N-1}\left(G(u)-\frac{N}{\beta} u\right) \xi d x=0
\end{aligned}
$$

On the other hand, by standard regularity results, it is clear that $u \in$ $C^{0}([0, \infty))$ and that in fact $u \in C^{2}$ on the set where the equation is not degenerate i.e. $\left\{x \in(0, \infty): u(x)>0\right.$ and $\left.\left(u^{m}\right)^{\prime}(x) \neq 0\right\}$. We shall show that the closure of this set coincides with the support of $u$. We can assume that $u^{m} \in C^{1}([0, \infty))$, because taking a sequence $\xi_{n}$ such that $\lim \xi_{n}(x)=1$ if $x \in\left[x_{0}-\varepsilon, x_{0}\right]$ and $\lim \xi_{n}(x)=0$ otherwise we have that

$$
\begin{aligned}
{\left[x^{N-1}\left|\left(u^{m}\right)^{\prime}\right|^{p-2}\left(u^{m}\right)^{\prime}\right]_{x_{0}-\varepsilon}^{x_{0}}+} & {\left[\frac{x^{N}}{\beta} u\right]_{x_{0}-\varepsilon}^{x_{0}}=} \\
& =-\int_{x_{0}-\varepsilon}^{x_{0}} x^{N-1}\left(G(u)-\frac{N}{\beta} u\right) d x .
\end{aligned}
$$

and so $\left|\left(u^{m}\right)^{\prime}\right|^{p-2}\left(u^{m}\right)^{\prime}\left(x_{0}\right)=0$ (the continuity at $x=0$ is similarly justified).

Ir consequence, by a solution of (14), (15), (16) we shall mean a function $u \in C^{0}([0, \infty))$ such that $u^{m} \in C^{l}([0, \infty)), u \geq 0 \quad(\not \equiv 0)$ and satisfies (16) and (17) for any smooth function $\xi$ with compact support in $[0, \infty)$.

Now we are in a condition to state our uniqueness results: 
Theorem 1. Assume that $N \geq 1, m>0, q>0, p>1$,

$$
m(p-1)>1
$$

and

$$
(p-1) m<q<(p-1) m+\frac{p}{N}
$$

Then there is at most one solution of problem (14), (15), (16). Moreover, this solution has compact support.

Theorem 2. The conclusion of Theorem 1 holds if we replace the assumption (18) of Theorem 1 by

$$
m(p-1)=1 .
$$

In this case, the solution is positive in $(0, \infty)$.

Before giving the proofs we shall make some remarks on the assumptions of both results. First of all we notice that the rcasonable assumption on the parameters $m$ and $p$ is $m(p-1) \geq 1$, because otherwise the parabolic equation (4) corresponds to a fast diffusion and solutions vanish after a finite time. On the other hand, it is natural to expect a different behaviour of solutions of (14), (15), (16) according to whether $m(p-1)$ is greatcr or equal to one. Indeed, the first case corresponds to slow diffusion, and the solutions of (4) have compact support for any value of $t$, although when $m(p-1)=1$ the solutions of $(4)$ are strictly positive in $\mathbb{R}^{N} \times(0, \infty)$. Finally the assumption (19) include the assumptions made in [1], [7] and [8] for the existence of very singular solutions. In that references it is also shown how boundary condition (16) implies the one given in (13).

\section{Proofs and auxiliary results}

The following Lemma collects several properties of solutions of (14), (15), (16).

Lemma 1. Assume $m(p-1) \geq I$ and condition (19). Let $u$ be any solution of (14), (15), (16). Then $u \in C^{0}$ and $u^{m} \in C^{1}$. Moreover

(i)

$$
\lim _{x \backslash 0} \frac{\left|\left(u^{m}\right)^{\prime}(x)\right|^{p-2}\left(u^{m}\right)^{\prime}(x)}{x}=-\frac{1}{N} G(u(0))
$$


(ii) $u(x) \leq M$ for any $x \geq 0$ with $M=\left(\frac{1}{q-1}\right)^{1 /(q-1)}$,

(iii) if $x_{0} \in[0, \infty)$ is such that $u\left(x_{0}\right)=0$ then $u(x)=0$ for any $x \geq x_{0}$,

(iv) $u(x)$ is monotone non-incresing in $(0, \infty)$ and $u^{\prime}(x)<0$ for any $x>0$ such that $u(x)>0$,

(v) if there exists a $x_{0} \in(0, \infty)$ such that supp $u=\left[0, x_{0}\right]$ then

$$
\lim _{x \uparrow x_{0}} \frac{\left|\left(u^{m}\right)^{\prime}(x)\right|^{p-1}}{u(x)}=\frac{x_{0}}{\beta}
$$

Remark. Condition (22) is equivalent to the diffcrential equation of the interface of the solution of the parabolic equation (4) which comes from the Darcy law (sce e.g. [7] for the case $p=2$ ).

Proof of Lemma 1: The regularity of $u$ has already beern proved in a previous remark, so we pass to consider the rest of the statcment.

Proof of (i): We multiply equation (14) by a smoth scquences of text functions $\xi_{n}(x)$ such that $\lim _{n} \xi_{n}(x)=1$ if $x \in[0, \epsilon]$ and $\lim _{n} \xi_{n}(x)=0$ otherwise, for some $\epsilon>0$. Intcgrating we have

$$
\begin{array}{r}
\left|\left(u^{m}\right)^{\prime}(\varepsilon)\right|^{p-2}\left(u^{m}\right)^{\prime}(\varepsilon)+\int_{0}^{\epsilon} \frac{N-1}{x}\left|\left(u^{m}\right)^{\prime}(x)\right|^{p-2}\left(u^{m_{2}}\right)^{\prime}(x) d x= \\
=-\frac{1}{\beta} \int_{0}^{\varepsilon} x u^{\prime}(x) d x-\int_{0}^{\epsilon} G(u(x)) d x
\end{array}
$$

Dividing by $\varepsilon$ and making $\varepsilon \rightarrow 0$ we obtain

$$
\begin{aligned}
\lim _{\varepsilon \downarrow 0}\left[\frac{\left|\left(u^{m}\right)^{\prime}(\varepsilon)\right|^{p-2}\left(u^{m}\right)^{\prime}(\varepsilon)}{\varepsilon}+\frac{N-1}{\varepsilon}\left[\left.\left(u^{m}\right)^{\prime}(\varepsilon)\right|^{p-2}\left(u^{m}\right)^{\prime}(\varepsilon)\right]=\right. \\
=-\operatorname{limrn}_{\varepsilon \downarrow 0} G(u(\varepsilon)),
\end{aligned}
$$

and therefore (i).

Proof of (ii): Assume by contrary that

$$
u\left(y_{0}\right)=\sup \{u(x): x \geq 0\}>M
$$

Then $u^{\prime}\left(y_{0}\right)=0$ and $\left(\left|\left(u^{m}\right)^{\prime}\right|^{p-2}\left(u^{m}\right)^{\prime}\right)^{\prime}\left(y_{0}\right) \leq 0$ (as $u^{m}$ also has his maxirmum in $\left.y_{0}\right)$. If $y_{0}>0$, from the differential cquation we deduce that.

$$
\left(\left|\left(u^{m}\right)^{\prime}\right|^{p-2}\left(u^{n u}\right)^{\prime}\right)^{\prime}\left(y_{0}\right)=-G\left(u\left(y_{0}\right)\right)>0 .
$$

If $y_{0}=0$, using (i) we find the same contradiction. Therefore $u \leq M$ on $[0, \infty)$. 
Proof of (iii): Again we shall argue by contradiction. Assume that (iii) is not true. Then it is easy to show that there exists $\varepsilon>0$ such that $u(x)>0$ and $u^{\prime}(x)>0$ on $\left(x_{0}, x_{0}+\varepsilon\right)$ (otherwise we can found a sequence $\left\{x_{n}\right\}$ of local minima of $u$ such that $x_{n} \rightarrow x_{0}$, which yields a contradiction with (14)).

Multiplying equation (14) by $x^{N-1}$ and integrating over $\left(x_{0}, x\right)$ with $x \in\left(x_{0}, x_{0}+c\right)$, we get

$$
\begin{aligned}
x^{N-1}\left|\left(u^{m}\right)^{\prime}(x)\right|^{p-1}+\int_{x_{U}}^{x} & \frac{s^{N}}{\beta} u^{\prime}(s) d s+ \\
& +\int_{x_{0}}^{x} \frac{s^{N-1}}{q-1} u(s) d s-\int_{x_{0}}^{x} s^{N-1} u^{q}(s) d s=0
\end{aligned}
$$

(we recall that. $u$ is regular in $\left(x_{0}, x\right)$ and $\left(u^{m}\right)^{\prime}\left(x_{0}\right)=0$ ). Taking a sequence of smooth test functions $\xi_{n}(s)$ in (14) such that $\lim _{\gamma_{2}} \xi_{\pi i}(s)=1$ if $s \in\left(x_{0}, x\right)$ (where $x_{0}<x<x_{0}+\varepsilon$ ) and $\lim _{n} \xi_{n}(s)=0$ otherwise, wc have that

$$
\begin{array}{r}
x^{N-1}\left|\left(u^{m L}\right)^{\prime}(x)\right|^{p-1}+\frac{x^{N}}{\beta} u(x)+\left(\frac{1}{q-1}-\frac{N}{\beta}\right) \int_{x_{0}}^{x} s^{N-1} u(s) d s= \\
=\int_{x_{0}}^{x} s^{N-1} u^{\prime}(s) d s
\end{array}
$$

(notice that $\left(u^{m}\right)^{\prime}\left(x_{0}\right)=0$ and $\left.\left(u^{m_{0}}\right)^{\prime}(x)>0\right)$. Using that $1 /(q-1)>$ $N / \beta$ we have

$$
\frac{x^{N}}{\beta} u(x) \leq \int_{x_{0}}^{x} s^{N-1} u^{q}(s) d s \leq \frac{1}{N}\left(x^{N}-x_{0}^{N}\right) u^{q}(x),
$$

or equivalently,

$$
\frac{1}{\beta} \leq \frac{1}{N} u^{q-1}(x)\left(1-\left(\frac{x_{0}}{x}\right)^{N}\right)
$$

Making now $x \rightarrow x_{0}$ we arrive to the inequality

$$
\frac{1}{\beta} \leq 0
$$

which is a contradiction.

Proof of (iv): Suppose that for some $x_{0}>0 \quad u^{\prime}\left(x_{0}\right)>0$, then by (i) there exists a $x_{1} \in\left(0, x_{0}\right)$ such that $u^{\prime}\left(x_{1}\right)=0$ and $\left(\left|\left(u^{m n}\right)^{\prime}\right|^{p-2}\left(u^{n t}\right)^{\prime}\right)^{\prime}\left(x_{1}\right)$ 
$\geq 0$. We also know by (iii) that $u\left(x_{1}\right)>0$. Arguing in the same way as in the proof of (i) we can show that

$$
\lim _{x \downarrow x_{1}} \frac{N\left|\left(u^{m}\right)^{\prime}(x)\right|^{p-2}\left(u^{m}\right)^{\prime}(x)}{x-x_{1}}=-\frac{1}{g-1}\left(u\left(x_{1}\right)-u^{q}\left(x_{1}\right)\right)<0
$$

(since $\left.0<u\left(x_{1}\right)<M\right)$. Then we arrive to a contradiction with the fact that $\left(\left|\left(u^{m}\right)^{\prime}\right|^{p-2}\left(u^{m}\right)^{\prime}\right)^{\prime}\left(x_{1}\right) \geq 0$. Thus $u^{\prime}(x) \leq 0$ for all $x \geq 0$. In fact the same argument shows that $u^{\prime}(x)<0$ for every $x>0$ where $0<u(x)<M$. Thus it only remains to exclude the case

$$
\begin{cases}u(x)=M & \text { if } x \in[0, a] \\ u(x)<M & \text { if } x \in(a,+\infty)\end{cases}
$$

for some $a>0$. We assert that there exists $\varepsilon>0$ such that for any $x \in(a, a+\varepsilon)$ we have

$$
u^{\prime}(x)<0
$$

and

$$
\begin{aligned}
-x^{N-1}\left|\left(u^{m}\right)^{\prime}(x)\right|^{\gamma-1}+ & \int_{a}^{x} \frac{s^{N}}{\theta} u^{\prime}(s) d s+ \\
& +\int_{a}^{x} \frac{s^{N-1}}{q-1} u(s) d s-\int_{a}^{x} s^{N-1} u^{q}(s) d s=0
\end{aligned}
$$

(the proof of these properties follow the same ideas used in the part (iii)). By integrating by parts we obtain

$$
\begin{aligned}
& x^{N-1}\left|\left(u^{m}\right)^{\prime}(x)\right|^{p-1}-\frac{x^{N}-a^{N}}{\beta} M+\frac{M-u(x)}{\beta} x^{N}= \\
& =\left(\frac{1}{q-1}-\frac{N}{\beta}\right) \int_{u}^{I} s^{N-1} u(s) d s-\int_{a}^{x} s^{N-1} u^{g}(s) d s .
\end{aligned}
$$

As $u$ is decreasing on $(a, a+\varepsilon)$ some elementary manipulation allows to obtain

$$
\begin{aligned}
-\frac{x^{N}-a^{N}}{\beta} & M+\frac{M-u(x)}{\beta} x^{N} \leq \\
\leq & \left(\frac{1}{q-1}-\frac{N}{\beta}\right) \frac{x^{N}-a^{N}}{N} M-\frac{x^{N}-a^{N}}{N}-u^{q}(x), \\
& \frac{M-u(x)}{\beta} x^{N} \leq \frac{x^{N}-a^{N}}{N}\left(\frac{M}{q-1}-u^{q}(x)\right)
\end{aligned}
$$


Dividing by $x^{N}(M-u(x))$ and letting $x \rightarrow a$ we arrive to the contradiction

$$
\frac{1}{\beta} \leq 0
$$

Thus, we have excluded the possibility $u=M$ on any interval $[0, a]$, and the proof of (iv) is now complete.

Proof of $(v)$ : Choose $\varepsilon>0$ such that $\varepsilon<x_{0}, u(x)>0$ and $u^{\prime}(x)<0$ in $x \in\left(x_{0}-\varepsilon, x_{0}\right)$. Then, as in part (iii), we obtain

$$
\begin{aligned}
& -x^{N-1}\left|\left(u^{m}\right)^{\prime}(x)\right|^{p-2}\left(u^{m}\right)^{\prime}(x)-\frac{x^{N}}{\beta} u(x)+ \\
& \quad+\left(\frac{1}{q-1}-\frac{N}{\beta}\right) \int_{x}^{x_{0}} s^{N-1} u(s) d s-\int_{x}^{x_{0}} s^{N-1} u^{q}(s) d s=0
\end{aligned}
$$

with $x \in\left(x_{0}-\varepsilon, x_{0}\right)$. Since $\left(u^{m}\right)^{\prime}(x)<0$ and $\frac{1}{q-1}>\frac{N}{\beta}$ we get

$$
\frac{\left|\left(u^{m}\right)^{\prime}\right|^{p-1}(x)}{u(x)} \leq \frac{x}{\beta}+\frac{\int_{x}^{x_{0}} s^{N-1} u^{q}(s) d s}{x^{N-1} u(x)}
$$

and

$$
\frac{\left|\left(u^{m}\right)^{\prime}\right|^{p-1}(x)}{u(x)}>\frac{x}{\beta}-\left(\frac{1}{q-1}-\frac{N}{\beta}\right) \frac{\int_{x}^{x_{0}} s^{N-1} u(s) d s}{x^{N-1} u(x)} .
$$

Letting $x \uparrow x_{0}$ in these two inequalities, we obtain at the limit

$$
\lim _{x \uparrow x_{0}} \frac{\left|\left(u^{m}\right)^{\prime}\right|^{p-1}(x)}{u(x)}=\frac{x_{0}}{\beta}
$$

Proof of Theorem 1: The first step is to introduce a change of unknown in such a way that the absorption term of the new equation be monotonically non-increasing. Let $v(x)$ defined by

$$
u(x)=v(x)^{\mu}
$$

If we take

$$
\mu=(p-1) /(m(p-1)-1)
$$

it is easy to see that $v$ satisfies (on the support of $v$ ) the equation (24)

$$
\left(\left|v^{\prime}\right|^{p-2} v^{\prime}\right)+\frac{N-1}{x}\left|v^{\prime}\right|^{p-2} v^{\prime}+\mu \frac{\left|v^{\prime}\right|^{p}}{v}+\frac{\mu x v^{\prime}}{\beta a v}+\frac{1}{(q-1) a}-\frac{v^{\mu(q-1)}}{a}=0
$$




$$
v \geq 0 \quad(\not \equiv 0) \text { in } \quad[0, \infty)
$$

$$
v^{\prime}(0)=0, \quad \lim _{x \rightarrow \infty} v(x)=0
$$

where

$$
a=(m \mu)^{(p-1)} .
$$

Now let $v_{1}$ and $v_{2}$ be two solutions of $(24),(25)$ and $(26)$. Let $x_{0} \in[0, \infty)$ be such that

$$
0<\left(v_{1}-v_{2}\right)\left(x_{0}\right)=\sup _{(0, \infty)}\left(v_{1}-v_{2}\right) \equiv h
$$

By comparing the value of

$$
\lim _{x \perp 0} \frac{\left|v_{i}^{\prime}(x)\right|^{p-2} v_{i}^{\prime}(x)}{x}
$$

for $i=1,2$, it is not difficult to sec that $x_{0}>0$.

Indeed, if $x_{0}=0$ we deduce, in the same way that in part (iii) of Lemma 1, that $v_{1}^{\prime}(x) \leq v_{2}^{\prime}(x)$ in $(0, \delta)$ and therefore

$$
\lim _{x \downarrow 0} \frac{\left|v_{1}^{\prime}(x)\right|^{p-2} v_{1}^{\prime}(x)}{x} \leq \lim _{x \downarrow 0} \frac{\left|v_{2}^{\prime}(x)\right|^{p-2} v_{2}^{\prime}(x)}{x}
$$

From this inequality ind the property of $v_{i}$

$$
\lim _{x \downarrow 0} \frac{\left|v_{i}^{\prime}(x)\right|^{p-2} v_{i}^{\prime}(x)}{x}=\frac{-1}{N a}\left(\frac{1}{q-1}-v_{i}^{(q-1) \mu}(0)\right)
$$

(sce part (i) of Lernma 1) we obtain $v_{1}(0) \leq v_{2}(0)$ and so the maximum of $v_{1}-v_{2}$ can not be attaint at $x_{0}=0$.

Assurne now that $x_{0}>0$ and $v_{2}\left(x_{0}\right)>0$. Obviously we also have that $v_{1}\left(x_{0}\right)>0$ because $h>0$. Then therc exists a constant $L>1$ such that

$$
\operatorname{minx}\left\{v_{1}(0)-v_{2}(0), v_{1}\left(z_{0}\right)-v_{2}\left(z_{0}\right)\right\}<\frac{v_{1}\left(x_{0}\right)-v_{2}\left(x_{0}\right)}{L}
$$

where $z_{0}>x_{0}$ is such that supp $v_{2}=\left[0, z_{0}\right]$. We also chose $k>0$ such that

$$
\max \left\{\frac{h}{L}, \frac{h}{2}\right\}<k<v_{1}\left(x_{0}\right)-v_{2}\left(x_{0}\right)=h
$$


Now, we shall first pay attention to the case $p \geq 2$. We multiply the equations of $v_{i}(i=1,2)$ by $x^{N-1} \xi$ with $\xi$ given by

$$
\xi=e^{p w}-1, \quad w=\left(v_{1}-v_{2}-k\right)^{+} .
$$

Integrating on $(0,+\infty)$ we have

$$
\begin{aligned}
& \int_{\left[\xi^{\prime} \neq 0\right]} x^{N-1}\left(\left|v_{1}^{\prime}\right|^{p-2} v_{1}^{\prime}-\left|v_{2}^{\prime}\right|^{p-2} v_{2}^{\prime}\right) \xi^{\prime}= \\
= & \int \mu x^{N-1}\left(\frac{\left|v_{1}^{\prime}\right|^{p}}{v_{1}}-\frac{\left|v_{2}^{\prime}\right|^{p}}{v_{2}}-\frac{v_{1}^{\mu(q-1)}}{\mu a}+\frac{v_{2}^{\mu(q-1)}}{\mu a}\right) \xi+\int \mu \frac{x^{N}}{\beta a}\left(\frac{v_{1}^{\prime}}{v_{1}}-\frac{v_{2}^{\prime}}{v_{2}}\right) \xi
\end{aligned}
$$

Using that $\xi^{\prime}=p w^{\prime} e^{p w}$ (where $w^{\prime}=v_{1}^{\prime}-v_{2}^{\prime}$ on $w>0$ and $w^{\prime}=0$ otherwise), the incquality (sec e.g. Díaz [2, p. 264])

(30) $\left(\left|\alpha_{1}\right|^{p-2} \alpha_{1}-\left|\alpha_{2}\right|^{p-2} \alpha_{2}\right)\left(\alpha_{1}-\alpha_{2}\right) \geq c\left|\alpha_{1}-\alpha_{2}\right|^{p} \quad \forall \alpha_{1}, \alpha_{2} \in \mathbb{R}^{+}$

and the fact that $\left(v_{2}-v_{1}\right) \xi \leq 0$ we deduce that

$$
\begin{aligned}
c \int x^{N-1}\left|w^{\prime}\right|^{p} e^{p w} d x \leq \mu \int \frac{x^{N-1}}{v_{1}} & \left(\left|v_{1}^{\prime}\right|^{p}-\left|v_{2}^{\prime}\right|^{p}\right) \xi d x+ \\
& +\frac{\mu}{\beta a} \int x^{N}\left(L n v_{1}-L n v_{2}\right)^{\prime} \xi d x,
\end{aligned}
$$

Moreover, applying the inequality (true for any $p>1$ )

$$
\left|\alpha_{1}\right|^{p}-\left|\alpha_{2}\right|^{p} \leq C\left|\alpha_{1}-\alpha_{2}\right| \quad \forall \alpha_{1}, \alpha_{2} \in \mathbb{R},
$$

(where $C$ denotes again a gencric constant and so it will denotes in the following), using that $v_{1}(x)>h / 2$ for any $x$ such that $w(x)>0$, and integrating by parts in the last integral we deduce that

$$
\begin{aligned}
c \int_{\left[w^{\prime} \neq 0\right]} x^{N-1}\left|w^{\prime}\right|^{p} e^{p w} d x \leq & \frac{2 \mu}{h} C \int_{\left[w^{\prime} \neq 0\right]} x^{N-1}\left|w^{\prime}\right|\left(e^{p n w}-1\right) d x+ \\
& +\frac{\mu}{\beta a} \int_{\left.\mid w^{\prime} \neq 0\right]} x^{N}\left(L n v_{1}-L n v_{2}\right)\left|\xi^{\prime}\right| d x .
\end{aligned}
$$

As the logarithm function is concave we have

$$
\begin{aligned}
C \int_{\left[w^{\prime} \neq 0\right]} x^{N-1}\left|w^{\prime}\right|^{p} e^{p w} d x \leq C \int_{\left[w^{\prime} \neq 0\right]} & x^{N-1}\left|w^{\prime}\right| e^{p w} d x+ \\
& +\frac{\mu}{\beta a} \int_{\left[w^{\prime} \neq 0\right]} \frac{v_{1}-v_{2}}{v_{2}} x^{N}\left|\xi^{\prime}\right| d x .
\end{aligned}
$$


But $\left[w^{\prime} \neq 0\right] \subseteq[w \neq 0]$ and from the choice of $w$ we deduce that there exists three positive constants $\delta_{1}, \delta_{2}, \delta_{3}$ such that if $x \in[0, \infty)$ satisfies that $w(x)>0$ then $x<\delta_{1}$ (because supp $v_{1}$ and supp $v_{2}$ are bounded), $x>\delta_{2}$ and $v_{2}(x)>\delta_{3}$ (as consequence of $(28),(29)$ and the monotonicity of $v_{1}$ ), we deduce

$$
C \int_{\left[w^{\prime} \neq 0\right]}\left|w^{\prime}\right|^{p} e^{p w} d x \leq \int_{\left[w^{\prime} \neq 0\right]}\left|w^{\prime}\right| e^{p w} d x
$$

or equivalently

$$
C \int_{\left[w^{\prime} \neq 0\right]}\left|\left(e^{w}\right)^{\prime}\right|^{p} \leq \int_{\left[w^{\prime} \neq 0\right]}\left|\left(e^{w}\right)^{\prime}\right| e^{(p-1) w} .
$$

Using Hölder inequality we have

$$
C\left(\int_{\left[w^{\prime} \neq 0\right]}\left|\left(e^{w}\right)^{\prime}\right|^{p}\right)^{\frac{p-1}{p}} \leq\left(\int_{\left[w^{\prime} \neq 0\right]} e^{p w}\right)^{\frac{p-1}{p}}
$$

and herice

$C\left(\int_{\left[w^{\prime} \neq 0\right]} e^{p w}\right)^{1 / p}+C\left(\int_{\left[w^{\prime} \neq 0\right]}\left|\left(e^{w}\right)^{\prime}\right|^{p}\right)^{1 / p} \leq(1+C)\left(\int_{\left[w^{\prime} \neq 0\right]} e^{p w}\right)^{1 / p}$.

So

$$
C\left\|e^{* 0}\right\|_{W^{1, p}\left(\left[w^{\prime} \neq 0\right]\right)} \leq\left\|e^{w}\right\|_{L^{p}\left(\left[w^{\prime} \neq 0\right)\right.}
$$

Assume now that $p<N$. Applying Sobolev and Hölder inequalities we obtain

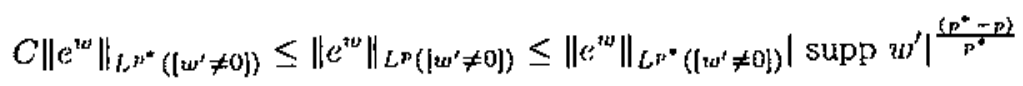

where $p^{*}=p N /(N-p)$. In particular

$$
\left|\operatorname{supp} w^{\prime}\right| \geq C>0
$$

In the case $p \geq N$ conclusion (32) is obtained from the Sobolev inequality by replaxing $p^{*}$ by any number greater than $p^{*}$. Since these inequalities are independent of $k$ they must hold as $k$ tends to $h$. That is, the function $v_{1}-v_{2}$ attain its supremum on a set of positive measure, where at the same time $\left(v_{1}-v_{2}\right)^{\prime}=0$, which is a contradiction with the inequality (32). 
In the case $1<p<2$ inequality (30) must be replaced by

$$
\left(\left|\alpha_{1}\right|^{p-2} \alpha_{1}-\left|\alpha_{2}\right|^{p-2} \alpha_{2}\right)\left(\alpha_{1}-\alpha_{2}\right) \geq C \frac{\left|\alpha_{1}-\alpha_{2}\right|^{2}}{\left(\left|\alpha_{1}\right|+\left|\alpha_{2}\right|\right)^{2-p}}
$$

(see e.g. Diaz $[2$, p. 264]). This justifies a change in the test function $\xi$ which now is taken as

$$
\xi=w=\left(v_{1}-v_{2}-k\right)^{+}
$$

Mutliplying the equations of $v_{i}$ by $x^{N-1} \xi$ and integrating on $(0, \infty)$ we have

$$
\begin{aligned}
C \int_{\left[w^{\prime} \neq 0\right]} x^{N-1} \frac{\left|w^{\prime}\right|^{2}}{\left(\left|v_{1}^{\prime}\right|+\left|v_{2}^{\prime}\right|\right)^{2-p}} d x \leq & \mu \int \frac{x^{N-1}}{v_{1}}\left(\left|v_{1}^{\prime}\right|^{p}-\left|v_{2}^{\prime}\right|^{p}\right) w d x+ \\
& +\frac{\mu}{\beta a} \int x^{N}\left(L n v_{1}-L n v_{2}\right)^{\prime} \xi d x
\end{aligned}
$$

But there exists $\delta_{1}>0$ such that $\left|v_{1}^{\prime}\right|+\left|x_{2}^{\prime}\right|>\delta_{1}$ on $\left[w^{\prime} \neq 0\right]$ (rectall part. (iv) of Lemma 1). Then it is easy to see that all the above arguments allow to obtain the inequality

$$
C\|w\|_{W 1,2\left(\left[w^{\prime} \neq 0\right]\right)} \leq\left\|e^{w}\right\|_{L^{2}\left(\left[w^{\prime} \neq 0\right]\right)}
$$

(instead of (31)) and so the conclusion follows.

Now we consider the last case: it is when the point $x_{0}$ is such that $v_{2}\left(x_{0}\right)=0$. We shall need a qualitative information which gives an additional information to part (v) of Lemma 1.

Lemma 2. Assume $m(p-1) \geq 1$ and (19). Let $u$ be any solution of (14), (15), (16). Then if supp $u=\left[0, x_{0}\right]$ we have

$$
\left|\left(u^{m}\right)^{\prime}\left(x_{1}\right)\right|^{p-1}<\frac{x_{1}}{\beta} u\left(x_{1}\right)
$$

for any $x_{1} \in\left(0, x_{0}\right)$.

Proof of Lemma 2: Integrating the equation (14) on $\left(0, x_{0}\right)$ we have

$$
\begin{aligned}
\int_{0}^{x_{0}}\left[\left(x^{N-1}\left|\left(u^{m}\right)^{\prime}\right|^{p-2}\left(u^{m}\right)^{\prime}\right]^{\prime}\right. & +\int_{0}^{x_{0}} \frac{1}{\beta}\left(x^{N} u\right)^{\prime}+ \\
& +\int_{0}^{x_{0}}\left(\left(\frac{1}{q-1}-\frac{N}{\beta}\right) x^{N-1} u-x^{N-1} u^{q}\right)=0
\end{aligned}
$$


but, has $\left(u^{m}\right)^{\prime}\left(x_{0}\right)=0$ and $u\left(x_{0}\right)=0$ we conclude that

$$
\int_{0}^{x_{0}} x^{N-1}\left(\left(\frac{1}{q-1}-\frac{N}{\beta}\right) u-u^{q}\right)=0 .
$$

From the monotonicity of $u$ (see part (iv) of Lemma 1) and the sign of $F(s)=\left(\frac{1}{q-1}-\frac{N}{\beta}\right) s-s^{q}$ (remember that $\left.\frac{1}{(q-1)}>\frac{N}{\beta}\right)$ we obtain

$$
\int_{0}^{x_{1}} x^{N-1}\left(\left(\frac{1}{q-1}-\frac{N}{\beta}\right) u-u^{q}\right)<0
$$

and

$$
\int_{x_{1}}^{x_{0}} x^{N-1}\left(\left(\frac{1}{q-1}-\frac{N}{\beta}\right) u-u^{q}\right)>0 .
$$

Integrating the equation (14) on $\left(0, x_{1}\right)$ we also have

$$
\begin{aligned}
\int_{0}^{x_{1}}\left(x^{N-1}\left|\left(u^{m}\right)^{\prime}\right|^{p-2}\left(u^{m}\right)^{\prime}\right)^{\prime} & +\int_{0}^{x_{1}} \frac{1}{\beta}\left(x^{N} u\right)^{\prime}+ \\
& +\int_{0}^{x_{1}}\left(\left(\frac{1}{q-1}-\frac{N}{\beta}\right) x^{N-1} u-x^{N-1} u^{q}\right)=0
\end{aligned}
$$

Thus (35) follows from this identity, (36), and the monotonicity of $u$ (part (iv) of Lemma 1).

Proof of Theorem 1 (Continuation): We was considering the case $v_{2}\left(x_{0}\right)=0$. As $v_{1}\left(x_{0}\right)>0$ we know that $v_{1}^{\prime}\left(x_{0}\right)<0$ (part (iv) of Lemma 1). So, necessarily supp $v_{2}=\left[0, x_{0}\right]$. We assume $p \geq 2$ (the case $1<p<2$ holds with obvious modifications). Let $y_{0}>x_{0}$ such that supp $v_{1}=[0, \%]$ (the compactness of the support of any solution is assumed here but it will be proved as a last step of the proof of this theorem). Using part ( $v$ ) of Lemma 1 we obtain

$$
\lim _{x \uparrow x_{0}} a\left|v_{2}^{\prime}(x)\right|^{p-1}=\frac{x_{0}}{\beta} .
$$

Thus function $v_{1}-v_{2}$ is not of class $C^{\mathrm{l}}((0, \infty))$ and so at its maximum $x_{0}$ we morly have the information

$$
\lim _{x \uparrow x_{0}} v_{1}^{\prime}(x) \geq \lim _{x x_{0}} v_{2}^{\prime}(x)
$$

Our arguments will be of a different type according the subcases

$$
\begin{aligned}
& \text { (a) } \lim _{x \uparrow x_{0}} v_{1}^{\prime}(x)=\lim _{x \uparrow r_{0}} v_{2}^{\prime}(x) \\
& \text { (b) } \lim _{x \uparrow x_{0}} v_{1}^{\prime}(x)>\lim _{x \uparrow x_{0}} v_{2}^{\prime}(x)
\end{aligned}
$$


Consider the subcase (a). From part (v) of Lernma 1 we obtain

$$
\lim _{x \rightarrow x_{0}} a\left|v_{2}^{\prime}(x)\right|^{p-1}=\frac{x_{0}}{\beta}=a\left|v_{1}^{\prime}\left(x_{0}\right)\right|^{p-1}
$$

On the other hand applying Lemma 2 we conclude that

$$
a\left|v_{1}^{\prime}\left(x_{0}\right)\right|^{p-1}<\frac{x_{0}}{\beta}
$$

which is a contradiction.

Now consider the subcase (b). Our main idea is to arguc in a similar way to the case $v_{2}\left(x_{0}\right)>0$. First of all we need to extend the equation of $v_{2}$ on $\left(x_{0},+\infty\right)$ : It is clear that $v_{2}$ is a wcak solution, in $\left(0, x_{0}\right) \cup\left(x_{0},+\infty\right)$, of the equation

$$
\left(\left|v_{2}^{\prime}\right|^{p-2} v_{2}^{\prime}\right)^{\prime}+\frac{N-1}{x}\left|v_{2}^{\prime}\right|^{p-2} v_{2}^{\prime}+\frac{1}{(q-1) a}-\frac{v_{2}^{\mu(q-1)}}{a}=B(x)
$$

where

$$
B(x)= \begin{cases}-\mu \frac{\left|v_{2}^{\prime}(x)\right|^{p}}{v_{2}(x)}-\frac{\mu x v_{2}^{\prime}(x)}{\beta a v_{2}(x)} & \text { if } x \in\left(0, x_{0}\right) \\ \frac{1}{(q-1) a} & \text { if } x \in\left(x_{0},+\infty\right) .\end{cases}
$$

Let us prove that $B \in L^{\infty}(0,+\infty)$. It is clear that we only noced to show that $\lim _{x \uparrow x_{0}} B(x)$ is finite. To do that we use one of the inequalities of the proof of part $(\mathrm{v})$ of Lemma 1 and we conclude

$$
\left|v_{2}^{\prime}(x)\right|^{\gamma^{\prime}-1}-\frac{x}{\beta a}>\frac{-1}{a}\left(\frac{1}{q-1}-\frac{N}{\beta}\right) \frac{\int_{x}^{x_{0}} s^{N-1} v_{2}^{\prime t}(s) d s}{x^{N-1} v_{2}^{\prime t}(x)}
$$

for any $x \in\left(0, x_{0}\right)$. Thus

$$
-\frac{\left|v_{2}^{\prime}(x)\right|^{p}}{v_{2}(x)}-\frac{x v_{2}^{\prime}(x)}{\beta a v_{2}(x)}<\frac{-v_{2}^{\prime}(x)}{a}\left(\frac{1}{q-1}-\frac{N}{\beta}\right) \frac{\int_{x}^{x_{0}} s^{N-1} v_{2}^{j}(s) d s}{x^{N-1} v_{2}^{t+1}(x)}
$$

on the interval $\left(0, x_{0}\right)$. By the l'Hôpital rule

$$
\begin{aligned}
& \lim _{x \nmid x_{0}} \frac{\int_{x}^{x_{0}} s^{N-1} v_{2}^{\prime \prime}(s) d s}{x^{N-1} v_{2}^{\mu+1}(x)}= \\
& =\lim _{\left.x \mid x_{0}\right)} \frac{-x^{N-1} v_{2}^{\prime \prime}(x)}{(N-1) x^{N-2} v_{2}^{\prime l+1}(x)+x^{N-1}(\mu+1) v_{2}^{\prime t}(x) v_{2}^{\prime}(x)}= \\
& =\lim _{x \uparrow x_{0}} \frac{-x}{(N-1) v_{2}(x)+x(\mu+1) v_{2}^{\prime}(x)}=\lim _{x \uparrow x_{0}} \frac{-1}{(\mu+1) v_{2}^{\prime}(x)}
\end{aligned}
$$


Using part. (v) of Lemma I we have in consequence

$\lim _{x \uparrow x_{0}} v_{2}^{\prime}(x)=-\left(\frac{x_{0}}{\beta a}\right)^{1 /(p-1)}, \quad \lim _{x \uparrow x_{0}} B(x) \leq \frac{1}{a(\mu+1)}\left(\frac{1}{q-1}-\frac{N}{\beta}\right)$.

On the other hand, if $x \in\left(0, x_{0}\right)$, by Lemma 2 we have

$$
B(x)=\frac{\mu v_{2}^{\prime}(x)}{a v_{2}(x)}\left[a\left|v_{2}^{\prime}(x)\right|^{p-1}-\frac{x}{\beta}\right]>0
$$

and so $\operatorname{limri}_{\substack{x_{1} \\ x_{0}}} B(x) \geq 0$.

From de notion of weak solution we have that

$$
\begin{aligned}
& -\int_{0}^{x_{0}} x^{N-1}\left(\left|v_{2}^{\prime}\right|^{p-2} v_{2}^{\prime}\right) \xi^{\prime}-\int_{x_{0}}^{+\infty} x^{N-1}\left(\left|v_{2}^{\prime}\right|^{p-2} v_{2}^{\prime}\right) \xi^{\prime}+ \\
& \quad+x_{0}^{N-1}\left(\lim _{x \uparrow x_{0}}\left|v_{2}^{\prime}(x)\right|^{p-2} v_{2}^{\prime}(x)\right) \xi\left(x_{0}\right)+ \\
& \quad+\int_{0}^{+\infty} x^{N-1}\left(\frac{1}{(q-1) a}-\frac{v_{2}^{\prime 2(q-1)}}{a}\right) \xi=\int_{0}^{+\infty} x^{N-1} B(x) \xi
\end{aligned}
$$

for any function $\xi \in W_{0}^{1, p}(0,+\infty)$. Now we chose $k>0$ such that

$$
v_{1}(0)-v_{2}(0)<k<v_{1}\left(x_{0}\right)-v_{2}\left(x_{0}\right)=v_{1}\left(x_{0}\right)=h \text {. }
$$

We take aggin

$$
\xi=e^{p \nu}-1, \quad w=\left(v_{1}-v_{2}-k\right)_{+} .
$$

Theri

$$
\begin{aligned}
& \int_{\left[\xi^{\prime} \neq 0\right]} x^{N-1}\left(\left|v_{1}^{\prime}\right|^{p-2} v_{1}^{\prime}-\left|v_{2}^{\prime}\right|^{p-2} v_{2}^{\prime}\right) \xi^{\prime}= \\
= & x_{0}^{N-1}\left(\lim _{x, \uparrow x_{0}}\left|v_{2}^{\prime}(x)\right|^{p-1}\right) \xi\left(x_{0}\right)+\int_{0}^{+\infty} x^{N-1}\left(\frac{-v_{1}^{\mu(q-1)}}{a}+\frac{v_{2}^{\mu(q-1)}}{a}\right) \xi+ \\
& +\int_{0}^{+\infty} \mu x^{N-1}\left(\frac{\left|v_{1}^{\prime}\right|^{p}}{v_{1}}+\frac{x v_{1}^{\prime}}{\beta a v_{1}}\right) \xi+\int_{0}^{+\infty} x^{N-1} B(x) \xi
\end{aligned}
$$

But (as $\xi(0)=0)$

$$
\xi\left(x_{0}\right)=\int_{0}^{x_{0}} \xi^{\prime}(s) d s \leq \int_{0}^{x_{0}}\left|\xi^{\prime}(s)\right| d s \leq \int_{\left[\xi^{\prime} \neq 0\right]}\left|\xi^{\prime}\right|
$$


On the other hand, using Lemma 2 we have

$$
\frac{\left|v_{1}^{\prime}\right|^{p}}{v_{1}}+\frac{x v_{1}^{\prime}}{\beta a v_{1}}=\frac{v_{1}^{\prime}}{v_{1}}\left(-\left|v_{1}^{\prime}\right|^{p-1}+\frac{x}{\beta a}\right)<0
$$

Then arguing as in the step $v_{2}\left(x_{0}\right)>0$ and using that $B \in L^{\infty}(0,+\infty)$ we obtain that there exists $C_{1}, C_{2}, C_{3}$ positive constants (not depending on $k$ ) such that

$$
C_{1} \int_{\left[w^{\prime} \neq 0\right]}\left|w^{\prime}\right|^{p} e^{p w} d x \leq C_{2} \int_{[w \neq 0]} e^{p w}+C_{3} \int_{\left[w^{\prime} \neq 0\right]}\left|\left(e^{w}\right)^{\prime}\right| e^{(p-1) w}
$$

Noting that

$$
w^{\prime}(x)= \begin{cases}v_{1}^{\prime}(x)-v_{2}^{\prime}(x) & \text { if } x \in(\operatorname{supp} w) \cap\left(0, x_{0}\right) \\ v_{1}^{\prime}(x) & \text { if } x \in(\operatorname{supp} w) \cap\left(x_{0}, y_{0}\right)\end{cases}
$$

that $v_{1}^{\prime}(x)<0$ on $\left(x_{0}, y_{0}\right)$ and that

$$
\lim _{x \nmid x_{0}}\left(v_{1}^{\prime}(x)-v_{2}^{\prime}(x)\right)>0
$$

we can chose $k$, closed enough to $h$, in order to have

$$
\operatorname{supp} w^{\prime}=\operatorname{supp} w
$$

Then, applying the Young inequality $a b \leq \varepsilon a^{p}+C_{E} b^{p /(p-1)}$ for $\varepsilon$ small enough $\left(\varepsilon<C_{1}\right)$ we get

$$
\left(C_{1}-\varepsilon\right) \int_{\left[w^{\prime} \neq 0\right]}\left|\left(e^{w}\right)^{\prime}\right|^{p} \leq\left(C_{2}+C_{3} C_{\varepsilon}\right) \int_{\left[w^{\prime} \neq 0\right]}\left|e^{w}\right|^{p}
$$

Now we are in the same situation than (31). Thus inequality (32) holds and we obtain the contradiction by making $k$ converging to $h$.

In order to complete the proof we must prove the compactness of the support of any solution of problem (14), (15), (16). For this surpose we shall define a supersolution of (14), (15), (16) with compact support. Let, $\phi$ be the function

$$
\phi(x)=\left(\left[C-x^{\circ}\right]^{+}\right)^{\mu} \quad \forall x \in[0, \infty),
$$

where $[v]^{+}=\max \{v, 0\}, a \in(1, p /(p-1))$ and $C$ is a positive constant to be determinate. After some elementary manipulations one can verify that $\left(\phi^{m}\right)^{\prime}(0)=0$ and that

$$
\left(\left|\left(\phi^{m}\right)^{\prime}\right|^{p-2}\left(\phi^{m}\right)^{\prime}\right)^{\prime}+\frac{N-1}{x}\left|\left(\phi^{m}\right)^{\prime}\right|^{p-2}\left(\phi^{m}\right)^{\prime}+\frac{1}{\beta} x \phi^{\prime}+\frac{1}{q-1} \phi-\phi^{q} \leq 0
$$


assumcd $C$ large enough. Hence $\phi$ is a supersolution of problem (14), (15), (16).

Arguing in the sarne way as in the proof of the uniqueness we can compare any solution of (14) with the supersolution $\phi$. Indeed: let $u$ be a solution and apply the previous change of variables to the functions $u$ and $\phi$. Then if we call

$$
v=u^{1 / \mu} \text { and } \psi=\phi^{1 / \mu}=\left[C-x^{\alpha}\right]^{+},
$$

$v$ is a solution of (24), (25), (26) and $\psi$ verifies

$$
\begin{aligned}
\left(\left|\psi^{\prime}\right|^{p-2} \psi^{\prime}\right)^{\prime}+\mu \frac{\left|\psi^{\prime}\right|^{p}}{\psi}+ & \frac{N-1}{x}\left|\psi^{\prime}\right|^{p-2} \psi^{\prime}+ \\
& +\frac{N}{\beta a} \frac{\psi^{\prime}}{\psi}+\frac{1}{q-1}-\frac{\psi^{\prime 2(q-1)}}{a} \leq 0 \operatorname{in}(0, C) .
\end{aligned}
$$

Now, proving that $\sup (v-\psi) \leq 0$ consists in repeating the same arguments as in the uniqueness proof, where now $v$ plays the role of $v_{1}$ and $\psi$ the one of $v_{2}$. Heree $\phi \geq u$ and since $\phi$ has a compact support, the same happens with $u$, and the proof of Theorem 1 is complet.

Proof of Theorem 2: As in the previous theorem, we introduce a change of unknown in order to arrive to a new equation with a monotone perturbation term. More precisely, let $v(x)$ defined by

$$
u(x)=e^{v(x)} \quad x>0
$$

(we suppose here that $u(x)>0$ as we shall prove in the last part of the Theorem). It is easy to see that $v$ satisfies

$$
\left(\left|v^{\prime}\right|^{p-2} y^{\prime}\right)^{\prime}+\left|v^{\prime}\right|^{p-2} v^{\prime}+\frac{x}{\beta} v^{\prime}+\frac{1}{q-1}-e^{(q-1) v}=0
$$

Now the uniqueness reduces to rcpeat the same arguments as before (cven in a easier way becanse the strict positivity of $v$ and the simplicity of the absorption and transport terms). In order to complete the proof of the Theorem 2 we just have to show that a solution $u$ of the problem (14), (15), (16) with $m(p-1)=1$ verifies $u(x)>0$ in $x[\in[0, \infty)$. Let suppose that there exists some $y_{0}$ such that $u\left(y_{0}\right)=0$. Then by Lemma 1 we know that supp $u=\left[0, x_{0}\right]$ for some $x_{0}>0$ and

$$
\lim _{x \mid x_{0}} \frac{\left|\left(z^{m}\right)^{\prime}(x)\right|^{1 / m}}{u(x)}=\frac{x_{0}}{\beta} .
$$


Let, us define the function $f(x)=\ln \left(x^{m}(x)\right)$ with $x \in\left[0, x_{0}\right)$, then we can write the previous limit as

$$
\lim _{x \uparrow x_{0}}\left|f^{\prime}(x)\right|=\left(\frac{x_{0}}{\beta}\right)^{m} .
$$

Since $\lim _{x \uparrow x_{0}} f(x)=-\infty$ and $f \in C^{1}\left(\left(0, x_{0}\right)\right)$ we arrive to a contradiction with (38), and the proof is concluded.

Remark. The idea of obtaining a contradiction via Sobolev inequalities was already used in Trudinger [10] (see also [4, Theorem 10.7]) to compare solutions of non-degenerate quasilinear elliptic problems. In that work the test function is defined as in the proof of Theorem 2. Finally we point out that our arguments can be also applied in order to obtain comparison results for solutions of more geveral equations, as for instance

$$
-\Delta_{p} u-\lambda \frac{|\nabla u|^{p}}{u}+B(x, u,|\nabla u|)+f(x, u)=0
$$

where $u \mapsto f(x, u)$ and $u \rightarrow B(x, u, \eta)$ are non-decreasing and $\eta \rightarrow$ $B(x, u, \eta)$ is Lipschitz continuous. In particular, this allows to generalize the uniqueness result of $[3]$.

Remark. Sirmultaneously to the completion of our work (which irnproves a previous version included in [9]) S. Kamin and L. Voron have communicated to us their work [6] in which they give a new proof of the existence of the very singular solution of the equation (5) as limnit of fundamental solutions satisfying $(7)$ when $c \rightarrow+\infty$. They also have a proof of the uniqueness of the very singular solution (i.c. a nonnegative not only self similar function satisfying (5)) and solutions of the parabolic equation (5). In this way they are giving an indirect proof of the uniquencss of $f$ for $p=2$ and $m>1$ arbitrary. It seerns that their arguments, jointly with some ideas of Kamin-Vazquez [5], may allow to give the uniqueness of the very singular solution in the class of solutions of (6) or even (4). In any case our arguments are of a different nature to those used in [6] and [5] and can be applied to other elliptic problems not necessarily related with the study of singular solutions of parabolic equations.

\section{References}

1. H. Brezis, L.A. Peletier and D. Terman, A very singular solution of the heat equation with absorption, Arch. Rat. Mech. Anal. 96 (1986). 
2. J.I. Dínz, "Nonlinear partial differential equations and free boundaries. Vol 1 Elliptic equations," Pitman Research Notes in Math. 106, Longman, 1985.

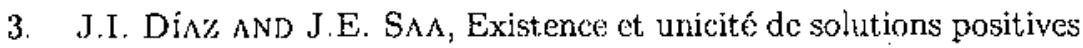
pour certaines équations elliptiques quasilineaires, CRAS Acad. Sci. Paris 305 (1987), 521-524.

4. D. Gilbarg and N.S. Trudinger, "Elliptic partial differential equations of second order," Springer-Verlag, 1983.

5. S. Kamin and J.L. VÁzQUEZ, Fundamental solutions and asymptotic behaviour for the $p$-Laplacian equation, Revista Matemática Iberoamericana 4 (1988), 339-354.

6. S. KAMIN ANI) L. Vekon, Existence and uniquencss of the very singular solutions of the porous media equations with absorption, Journal d'Analyse Mathématique 51 (1988), $245 \div 258$.

7. L.A. PEleTisR AND D. TERMAN, A very singular solution of the porous media equation with absorption, J. Diff. Equ. 65 (1985), $396-410$.

8. L.A. Peletier AND J. WANG, A very singular solution of a degenerate diffusion equation with absorption, Transactions of the AMS 307 (1988), 2813-2826.

3. J.E. SAA, Doctoral Thesis at the University Compluterse of Madrid, Novenber, 1988.

10. N.S. Trudinger, On the comparison principle for quasilinear divergence structure equations, Arch. Rational Mech. Anal. 57 (1973), $128-133$.

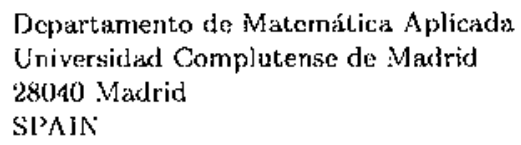

Primera versió rebuda el 10 de Setembre de 1990 , darrera versió rebuda el 23 d'Octubre de 1991 